\title{
Studying the Creation of Design Artifacts
}

\author{
Davis, Christopher J. ; Hevner, Ala n R. ; Weber, Barbara
}

Published in:
Information Systems and Neuroscience

Link to article, DOI:

10.1007/978-3-319-41402-7_15

Publication date:

2016

Document Version

Peer reviewed version

Link back to DTU Orbit

Citation (APA):

Davis, C. J., Hevner, A. N. R., \& Weber, B. (2016). Studying the Creation of Design Artifacts. In Information Systems and Neuroscience: Gmunden Retreat on NeurolS 2016 (pp. 115-122). Springer. Lecture Notes in Information Systems and Organisation Vol. 16 https://doi.org/10.1007/978-3-319-41402-7_15

\section{General rights}

Copyright and moral rights for the publications made accessible in the public portal are retained by the authors and/or other copyright owners and it is a condition of accessing publications that users recognise and abide by the legal requirements associated with these rights.

- Users may download and print one copy of any publication from the public portal for the purpose of private study or research.

- You may not further distribute the material or use it for any profit-making activity or commercial gain

- You may freely distribute the URL identifying the publication in the public portal

If you believe that this document breaches copyright please contact us providing details, and we will remove access to the work immediately and investigate your claim 


\title{
Studying the Creation of Design Artifacts
}

\author{
Christopher J. Davis ${ }^{1}$, Alan R. Hevner ${ }^{2}$ and Barbara Weber ${ }^{3,4}$ \\ ${ }^{1}$ University of South Florida, Saint Petersburg, FL USA \\ davisc@mail.usf.edu \\ ${ }^{2}$ University of South Florida, Tampa, FL USA \\ ahevner@usf.edu \\ ${ }^{3}$ Technical University of Denmark, Copenhagen, Denmark \\ bweb@dtu.dk \\ ${ }^{4}$ University of Innsbruck, Innsbruck, Austria
}

\begin{abstract}
As software and information systems (IS) increase in functional sophistication, perceptions of IS quality are changing. Moving beyond issues of performance efficiency, essential qualities such as fitness for purpose, sustainability, and overall effectiveness become more complex. Creating software and information systems represents a highly interconnected locus in which both the generative processes of building design artifacts and articulating constructs used to evaluate their quality take place. We address this interconnectedness with an extended process-oriented research design enabling multi-modal neurophysiological data analyses. We posit that our research will provide more comprehensive assessments of the efficacy of design processes and the evaluation of the qualities of the resulting design artifacts.
\end{abstract}

Keywords: IS Design · Creating Design Artifacts · Personal Construct Psychology (PCT) · Event-related potential (ERP) · Eye-tracking · Eye fixation related potential (EFRP) · Electroencephalography (EEG) · Interaction Logging - Repertory Grid Analysis (RGA)

\section{Introduction}

Creative design activities are central to all applied engineering disciplines. The information systems (IS) field since its advent has the principal objective of designing, building, and evaluating systems to solve complex business problems. IS as composed of inherently mutable and adaptable hardware, software, telecommunications, and human interfaces provide many unique and challenging design problems that call for new, creative ideas and discovery. IS artifacts are implemented within an application context for the purpose of improving the effectiveness and efficiency of that context. The utility of the artifact and the characteristics of the application - its work systems, its people, and its development and implementation methodologies - together determine the extent to which that purpose is achieved. Researchers produce new ideas that enhance generative capacity 
[1] and improve the ability of human organizations to adapt and succeed in the presence of changing environments. These generative ideas are then communicated as knowledge to the various IS communities [2].

The IS design environment is characterized by significant (and increasingly complex) generative opportunities presented by the diversification of rapidly evolving technologies in terms of development focus and medium (e.g. 'wrappers' for legacy systems; XaaS; cloud/virtualization; mobile apps) and agility (e.g. speed of creation and deployment). Creative design activities are supported by a plethora of representational methods and tools. With all of this richness of creative design opportunities and enabling creative infrastructures, IS researchers still struggle to understand the process of creating artifacts in IS design - and how to measure their quality. Thus, we pose the research question: Has the diversification of functionalities, development environments, tools (e.g. representational languages) etc. expanded the range of criteria that are used to guide the creative design process and improved our ability to judge the quality of design artifacts? In other words, have new understandings and theories of creating design artifacts emerged in response to advances in design processes and tool evolution and their innovative use? We posit that the answer is Yes. The remainder of this paper presents a comprehensive research design and protocols that enable emergent characteristics of design quality to be identified and articulated, providing insight into the process of creating design artifacts. We triangulate a rich array of previously inchoate empirical data sources to rigorously address the research question set out above. This paper extends research proposed by Davis and Hevner [3] on how designers employ visual syntax in the process of creating IS design artifacts.

\section{The Creation of Design Artifacts}

Design is both a process and a product. It describes the world as acted upon (processes) and the world as sensed (artifacts). In this research the phenomena of interest are the creation process and the quality assessment of the created design artifacts. As we have noted previously [4] this view of design supports a problem-solving paradigm that continuously shifts perspective between design processes and designed artifacts for the same complex problem. The design process is a sequence of activities tapping a range of expertise that produces an innovative product (i.e., the design artifact). The perceived qualities of the artifact enable evaluation which provides feedback, leading to a better understanding of the design challenge and, in turn, to improvement of the qualities of both the product and the design process.

Hevner, Davis, Collins, and Gill [4] propose a $2 \times 2$ model of the design process from the perspective of neuroscience. The $\mathrm{x}$-axis distinguishes the External (Task) Environment from the Internal (Cognitive) Environment; the y-axis separates the Problem Space from the Solution Space. In this research, we focus on the iterations of observation and generation of candidate designs that advance the design process from the upper-right internal problem space to the lower-right internal solution space, creating new, effective candidate solutions in response to the requirements in the problem space - the process of creation. This flow enables creative design events - deci- 
sions and actions - to be captured and analyzed in real-time. The form and immediacy of the post-task analyses articulate the constructs that guide design events and evaluation of the quality of the candidate solution artifact. Our research framework overcomes the limitations of instruments such as the Remote Associates Test and the Alternative Uses Task: their emphasis on comprehension limits their capacity to accommodate emerging, i.e. not yet fully known, cognitive constructs.

The specific questions addressed here concern the characteristics used to guide the creation of conceptual models (e.g. UML diagrams). We argue that this generative component of design differs substantially from the primarily comprehension based tasks that have guided prior research (e.g. [5-8]). Our goal is to identify the constructs used by designers to articulate the qualities that guide creating and evaluating designs. Our prior work [9] shows that the combination of neurophysiological data - particularly those elicited using the EFRP and ERP protocols - and interaction logs provide an authoritative basis to identify design decisions and the quality characteristics driving them. In this paper, we present a comprehensive research design and protocol, in Sections 3 and 4, respectively, that enable neurophysiological data and interaction logs to be synchronized. This composite data set provides the basis for eliciting the constructs undergirding design choices and design actions, i.e., interactions with the design platform.

\section{Research Design}

The build and evaluate cycles of design are typically iterated a number of times before the final (use) artifact is released into an application context for further testing and assessment through field study. During the process of creating and refining design artifacts the researcher must be cognizant that both the design process and the design artifact evolve as the research progresses. Kelly's [10] Personal Construct Theory (PCT) accommodates the socio-cognitive milieu that characterizes the co-evolution of the process of creating design artifacts and the constructs that guide their evaluation.

PCT [10] was developed as a framework for understanding how people made sense of the world around them. Kelly argued that people act as 'lay epistemologists' [11] in their attempts to order and interpret their experiences, categorizing and discriminating between them. In this way, individuals develop systems of interrelated personal constructs that enable them to anticipate the consequences of their own actions - and interpret the actions of others. PCT allows researchers to tap into the mental models used by individual designers to frame and articulate the world as acted upon and the world as sensed. PCT is particularly well suited to our phenomenon of interest - the qualities guiding the process of creating and evaluating design artifacts.

PCT is translated into practice using a cognitive mapping technique called Repertory Grid Analysis (RGA). Individuals' perceptions of similarity and difference are elicited, tapping into their theories of how the world operates. Use in its 'minimum context' form exploits the potential of RGA to directly tap designers' perceptions. This maintains the integrity of "the complex interconnectedness of the self and its social surroundings" [12, p345] providing two substantial benefits. First, it accommo- 
dates a wide range of empirical data types during elicitation of perceptions of the design process and product, including intermediate versions of the design artifact in the processes of building artifacts and evaluating progress. This enables design decisions that drive evolution of the design artifact from one version to another to be articulated directly by their creator. Second, relying on PCT to guide analysis of these constructs provides more ready and authentic construing of construction processes by members of the design community that we study. This responds directly to calls from the NeuroIS community for robustness and rigor in the application of neuro techniques [13]. The need for a more a comprehensive research protocol in order to accommodate the longer-term (multi-episodic) series of cognitive 'events' that generative tasks associated with creating software design artifacts entail is highlighted by Weber et al. [9]. The protocol set out below strives to maintain the 'interconnectedness' of designer and design [12] in a workplace setting, significantly increasing the immersion of the research into the design context - and authenticity of the analyses.

\section{$4 \quad$ Research Protocol}

Figure 1 outlines the research protocol and shows a three-phase 'immersion' into the complex interconnectedness of design environments. In order to exploit the capacities of PCT and RGA, identification of cognitive 'events' indicating design decisions and reconstruction of the design artifact before and after the design decision are required. In Phase 1 the designer works on a design task, i.e., creates a design artifact using a design platform. During this phase we rely on multi-modal data collection: we conduct neuro-physiological measurements, collect EEG data and instrument the design platform to simultaneously gather data on the design activities, i.e., interactions with the design platform. Data are synchronized using timestamps.

In Phase 2 we address the transition of designers from creating to evaluating: the events that interconnect designer and design. The stream of neurophysiological data is used to identify relevant cognitive events, i.e., the points in time when decisions took place, using the ERP and EFRP protocols with the EEG data. Timestamps are used to extract an intermediate model associated with each cognitive event from the stream of design activities [14-17] providing a secure basis for data triangulation.

In Phase 3 a series of steps is used to elicit personal constructs. Firstly, intermediate model versions are presented to the designer three at a time: their similarities and differences are used to articulate the 'poles' of a dimension that differentiate them. The poles provide the labels for a row in the repertory grid. This step is repeated until all version combinations have been exhausted and/or repetition occurs. The columns of the grid are labelled using the (numbered) model versions - the design task 'elements' that the grid represents. The rows are also numbered, 1 indicating the left-side label and 5 indicating the right-side label, providing a simple Likert scale for later use.

The (subject) designer completes the next step alone: working one row at a time, the designer rates each model version (element) using the 1-5 scale. The completed grid is analyzed using two-dimensional (spaced-focused) cluster analysis to re-order 
the grid and add dendograms (crow's feet) to illustrate the linkages or clustering of the constructs.

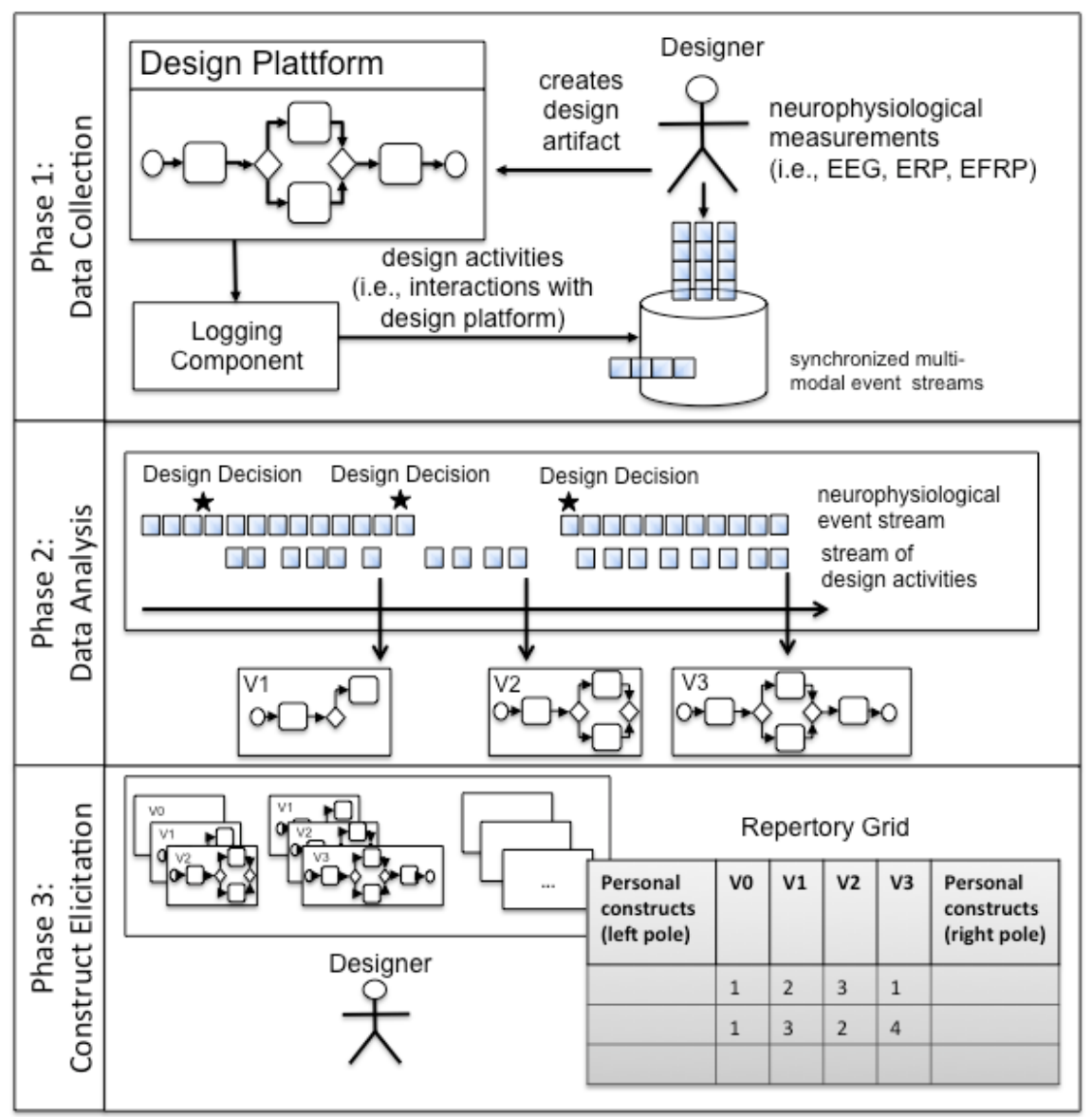

Figure 1 Immersive Analysis Protocol

The final step uses a protocol called 'talkback' to guide reflection on the spacedfocused grid. The designer articulates the qualities that characterize the most closely linked constructs: these are recorded by the researcher. This interview phase is iterative, moving from the smallest clusters 'outwards' to articulate more universal constructs. The system of inter-related constructs that represent the subject designer's view of the essential characteristics of design is captured in this phase.

The protocol balances the subject-centricity offered by PCT - essential to elicit the constructs guiding the process of creating and evaluating design artifacts - with the rigor of the detection of intermediate versions of the design artifact from interaction logs, ERP, EFRP and RGA protocols. There are three specific benefits: (i) accommodation of a range of complementary data types; (ii) maintenance of data cohesion and 'interconnectedness' by eliciting and analyzing them in a well-bounded experimental 
setting (i.e. generative design in the world of the designer); and (iii) exploiting the capacity of minimum context RGA and the talkback protocol to provide a medium or 'conversational technology' [18] - that enables expert subjects themselves to interpolate the disparate data types generated during the experiment. This adds coherence and accommodates the longevity and cumulative nature of design, enabling investigation of design decisions and model interactions in near to real-time. Further, the RGA protocols mitigate limitations of techniques such as talk-aloud, removing the risks associated with loss of recall and interference with neurophysiological data gathering. The re-connection of designer and design process through RGA overcomes these limitations, enabling disparate data types to be orchestrated in real-time to provide new insights into the design process as it unfolds in the work setting

We anticipate that prompt use of RGA to re-immerse the subject in their design experience will enable the constructs used to create and evaluate design artifacts to be readily and reliably elicited. Prior use of these protocols has been revelatory to both researcher and subjects, identifying constructs that had previously neither been anticipated or articulated [11]. Such emergent constructs will, we envisage, enable the range of criteria used to assess the utility and other qualities of design processes and artifacts to be significantly expanded.

\section{$5 \quad$ Discussion and Future Directions}

The research design is novel: the phenomena of interest - the design processes and the qualities of the artifacts they generate - have proved elusive. This novelty is complemented by focus on the creative transitions between building and evaluating, offering new insight into the qualities that drive the processes of creating and selecting candidate design artifacts.

The combination of process-orientation with multi-modal data gathering has significant benefits and implications for both theory and practice. The immersive analysis substantially extends and enriches assessment of design processes and artifacts. Fuller understanding of the qualities that guide design decisions during the creation of design artifacts will also provide useful advice for practicing designers and trainers.

PCT maintains the interconnectedness of designer and design and moves towards a more tractable definition of creation - the 'process of creating design artifacts', overcoming the limitations of prior neuro and NeuroIS studies [13, 14]. For instance, research into conceptual modeling is dominated by comprehension-oriented tasks (e.g., [19-22]) rather than conceptual modeling tasks (e.g., [23]) and research settings applying neuro-physiological tools favor static diagrams over (dynamic) processes (e.g., [5-8]). Moreover, in a more general context, most of the studies applying neurophysiological tools in IS are stimulus-response tasks rather than generative - addressing short-term experiments (episodes) rather than longer-term processes [14, p. 107].

The paper also serves as an invitation to the NeuroIS community to provide feedback on the feasibility and viability of the research design and to explore collaborative means of achieving the critical mass of resources required to bring it to fruition. 


\section{References}

1. Avital, M. and Te'eni, D. (2009) From generative fit to generative capacity: Exploring an emerging dimension of information systems design and task performance. Information Systems Journal, 19(4), 345-367.

2. Gregor, S. and Hevner, A. (2013) Positioning and presenting design science research for maximum impact, Management Information Systems Quarterly, 37(2).

3. Davis, C. and Hevner, A. (2015) Neurophysiological Analysis of Visual Syntax in Design. Information Systems and Neuroscience: Gmunden Retreat on NeuroIS, Springer, Gmunden, Austria, pp. 99-106.

4. Hevner, A., Davis, C., Collins, R. and Gill, T. (2014) A NeuroDesign Model for IS Research. Informing Science: The International Journal of an Emerging Transdiscipline 17, pp. 103-132.

5. Mendling, J., Strembeck, M., Recker, J. (2012) Factors of process model comprehension - findings from a series of experiments. Decision Support Systems 53(1), 195-206.

6. Figl, K., Recker, J., Mendling, J. (2013) A study on the effects of routing symbol design on process model comprehension. Decision Support Systems 54(2), 1104-1118.

7. Figl, K., Mendling, J., Strembeck, M. (2013) The influence of notational deficiencies on process model comprehension. Journal of the Association for Information Systems, 14(6), Article 1

8. Recker, J., Reijers, H.A., Van de Wouw, S.G. (2014) Process model comprehension: the effects of cognitive abilities, learning style, and strategy. Communications of the Association for Information Systems 34(9), 199-222.

9. Weber, B., Pinggera, J., Neurater, M., Zugal, S., Martini, M., Furtner, M., Sachse, P. and Schnitzer, D. (2016) Fixation Patterns During Process Model Creation: Initial Steps Toward Neuro-adaptive Process Modeling Environments Proceedings of the $49^{\text {th }}$ Hawai' $i$ International Conference on Systems Sciences (HICSS49) Kauai, Hawai'i, pp. 600-609.

10. Kelly, G. (1955) The Psychology of Personal Constructs. Norton, London, UK

11. Davis, C. and Hufnagel, E. (2007) Through the Eyes of Experts: A Socio-cognitive Perspective on the Automation of Fingerprint Work. MIS Quarterly 31(4), pp. 681-703.

12. Neimeyer, G. and Neimeyer, R. (1985) Relational Trajectories: A Personal Construct Contribution. Journal of Social and Personal Relationships 2, pp. 325-349.

13. Riedl, R., Davis, F. and Hevner, A. (2014) Towards a NeuroIS Research Methodology: Intensifying the Discussion on Methods, Tools and Measurement. Journal of the Association for Information Systems 15, pp. i-xxxv.

14. Riedl, R., Léger, J-P., Fundamentals of NeuroIS (2016). Information Systems and the Brain. Studies in Neuroscience, Psychology, and Behavioral Economics. Berlin, Heidelberg, Springer.

15. Müller-Putz, G., Riedl, R. and Wriessnegger, S. (2015) Electroencephalography (EEG) as a Research Tool in the Information Systems Discipline: Foundations, Measurement and Applications. Communications of the Association of Information Systems 37(46) pp 911948.

16. Pinggera, J., Zugal, S., Weidlich, M., Fahland, D., Weber, B., Mendling, J. and Reijers, H. (2012). Tracing the Process of Process Modeling with Modeling Phase Diagrams. In: Proc. ER-BPM '11, pp. 370-382.

17. Pinggera, J., Zugal, S. and Weber, B. (2010). Investigating the Process of Process Modeling with Cheetah Experimental Platform. In: Proc. ER-POIS '10, pp. 13-18.

18. Thomas, L. and Harri-Augstein, S. (1985) Self-Organized Learning: Foundations of a Conversational Science for Psychology. Routledge \& Kegan-Paul, plc London, UK. 
19. Matsuo, N., Ohkita, Y., Tomita, Y., Honda, S. and Matsunaga, K. (2001) Estimation of an Unexpected-Overlooking Error by Means of the Single Eye Fixation Related Potential Analysis with Wavelet Transform Filter. International Journal of Psychophysiology 40(3), pp. 195-200.

20. Takeda, Y., Sugai, M. and Yagi, A. (2001) Eye Fixation Potentials in a Proof-reading Task. International Journal of Psychophysiology 40(3), pp. 181-186.

21. vom Brocke, J., Riedl, R. and Léger, P. (2013) Application Strategies for Neuroscience in Information Systems Design Science Research. Journal of Computer Information Systems 53(3), pp. 1-13.

22. Recker, J., Safrudin, N., Rosemann, M. (2012) How novices design business processes. Inf. Syst. 37 (6), 557-573.

23. Hungerford, B., Hevner, A., and Collins, R. (2004) Reviewing Software Diagrams: A Cognitive Study. IEEE Transactions on Software Engineering 30(2), pp. 82-96. 